\title{
Philonsorbonne
}

$7 \mid 2013$

Année 2012-2013

\section{Le problème des prédictions dans les sciences expérimentales}

Gauvain LECONTE

\section{OpenEdition}

1 Journals

Édition électronique

URL : https://journals.openedition.org/philonsorbonne/495

DOI : 10.4000/philonsorbonne.495

ISSN : 2270-7336

Éditeur

Publications de la Sorbonne

Édition imprimée

Date de publication : 15 novembre 2013

Pagination : 81-99

ISSN : 1255-183X

\section{Référence électronique}

Gauvain LECONTE, «Le problème des prédictions dans les sciences expérimentales », Philonsorbonne [En ligne], 7 | 2013, mis en ligne le 18 décembre 2013, consulté le 08 juin 2021. URL : http:// journals.openedition.org/philonsorbonne/495; DOI : https://doi.org/10.4000/philonsorbonne.495 


\title{
Le problème des prédictions dans les sciences expérimentales
}

\author{
Gauvain Leconte
}

\begin{abstract}
Il est une circonstance où se marque, avec une netteté particulière, notre croyance au caractère naturel d'une classification théorique; cette circonstance se présente lorsque nous demandons à la théorie de nous annoncer les résultats d'une expérience avant que cette expérience n'ait été réalisée, lorsque nous lui enjoignons cet ordre audacieux : "Prophétise-nous ».
\end{abstract}

Pierre Duhem ${ }^{(*)}$

Si, sans perturber le système en aucune façon, nous pouvons prédire avec certitude (c'est-à-dire avec une probabilité égale à 1) la valeur d'une grandeur physique, alors il existe un élément de la réalité physique correspondant à cette grandeur physique.

Einstein, Podolsky et Rosen ${ }^{(* *)}$

\section{Introduction}

Parmi les succès scientifiques, le plus impressionnant et le plus recherché est sûrement la réussite prédictive. Thalès a prédit une éclipse, Newton le retour de la comète de Halley et Einstein la courbure des rayons lumineux par le Soleil: l'histoire des progrès scientifiques est souvent l'histoire des prédictions réussies et si prédire n'est probablement pas le seul objectif de l'activité scientifique, c'est un objectif qui fait consensus. Personne ne nierait qu'une théorie dotée d'une forte capacité prédictive - qui prédit de nombreux phénomènes différents avec précision - est toujours préférable, toutes choses égales par ailleurs, à une théorie ayant une plus faible capacité prédictive.

Mais les prédictions réussies ne sont pas qu'un moyen de confirmer et de choisir parmi des théories concurrentes ; comme le prouvent les citations de Duhem et d'Einstein placées en exergue ci-dessus, une prédiction réussie est bien souvent, pour les scientifiques comme pour les épistémologues,

(*). P. Duhem, La Théorie physique, son objet, sa structure, 1914 ( $2^{\mathrm{e}}$ édition), Paris, Vrin, p. 37.

(**). Einstein, Podolsky et Rosen, «Can Quantum-Mechanical Description of Physical Reality be Considered Complete?», Physical Review, vol. XLVII (1935), traduction française F. Balibar, Albert Einstein CEuvres choisies, t. I «Quanta », Paris, Éditions du Seuil et du CNRS, 1989, p. 225. 
considérée comme une preuve de la réalité des entités, des grandeurs et des structures supposées par une théorie. La réussite prédictive semble être le seul moyen de vérifier, par des moyens accessibles aux scientifiques, si une théorie est vraie ou approximativement vraie, c'est-à-dire si elle est en adéquation non seulement avec le monde observable mais aussi avec la réalité non-observable.

Mais sait-on réellement comment nous réalisons des prédictions, et ce que l'on appelle la capacité prédictive d'une théorie ? Ce problème concernant spécifiquement la nature des prédictions n'a attiré que récemment l'intérêt des épistémologues, car pendant longtemps cette notion paraissait évidente et non problématique. On peut ainsi distinguer ce problème spécifique des prédictions scientifiques d'autres problèmes qui font intervenir cette notion mais ne la concernent pas directement, comme les questions posées par les conceptions dites instrumentalistes : « Le but de l'activité scientifique n'est-il que de faire des prédictions? », «Les théories ne sont-elles que des outils dénuées de toute valeur de vérité permettant de réaliser des prédictions? ».

Comment s'est fait ce passage d'un ensemble de problèmes traditionnels à ce problème spécifique? Il a fallu attendre que deux grands débats de philosophie des sciences convergent vers le problème de la nature des prédictions pour que l'on réévalue la définition de cette notion fondamentale. Ces deux débats sont ceux entourant l'élaboration d'une théorie de la confirmation empirique et la thèse dite du réalisme scientifique.

Le but de cet article est de montrer d'abord comment ces deux domaines ont donné naissance et modelé le problème spécifique des prédictions et d'examiner ensuite une des solutions à ce problème.

\section{Les deux racines du problème spécifique des prédictions scientifiques}

\section{Problèmes du prédictivisme}

On distingue habituellement, en philosophie des sciences, les prédictions des accommodations de la manière suivante :

- si un phénomène $\mathrm{E}$ est connu avant la formulation de la théorie $\mathrm{T}$ qui l'explique on dit que $\mathrm{T}$ accommode $\mathrm{E}$;

- si un phénomène $\mathrm{E}$ n'est observé qu'après la formulation de la théorie $\mathrm{T}$ à partir duquel on l'infère on dit que $\mathrm{T}$ prédit $\mathrm{E}$.

Le fait que les lois et les théories les plus confirmées et couronnées de succès, comme celles de Newton et d'Einstein en physique ou la classification des éléments de Mendeleïev en chimie, aient réalisé des prédictions réussies semble montrer que les prédictions comptent plus que 
les accommodations. Mendeleïev par exemple obtint la médaille Davy ${ }^{1}$ en 1882, soit quelques années à peine après la découverte des nouveaux éléments Gallium et Scandium, en 1875 et 1879 respectivement, deux éléments dont il avait prédit l'existence, les propriétés et la manière dont ils seraient découverts en 1871. Apparemment, comme l'affirme Peter Lipton, après ces découvertes «les soixante accommodations des éléments déjà connus firent pâle figure face à deux prédictions $»^{2}$. Cette thèse consistant à affirmer qu'un phénomène $\mathrm{E}$ confirme plus une théorie ou une hypothèse s'il est prédit par elle que s'il est accommodé se nomme le prédictivisme.

Mais si l'on distingue ainsi prédictions et accommodations, le prédictivisme est difficilement soutenable d'un point de vue logique et très imprécis, voire faux, d'un point de vue historique.

D'un point de vue logique, la théorie de la confirmation a été définie par Hempel comme «la quête de critères objectifs déterminant quand et - si possible - à quel degré une hypothèse $\mathrm{H}$ peut être dite confirmée par un corps de preuves $\mathrm{E} »^{3}$. Comme le remarque Musgrave, dont l'article de 1974 est souvent considéré comme le point de départ de la littérature moderne sur les prédictions scientifiques, cette théorie classique de la confirmation est « logiquement pure " c'est-à-dire que pour «décider si une hypothèse $\mathrm{H}$ est ou non confirmée par la preuve $\mathrm{E}$, on doit considérer uniquement les énoncés $\mathrm{H}$ et $\mathrm{E}$, et les relations logiques entre eux $»{ }^{4}$.

Cette conception classique de la confirmation rencontre de nombreux problèmes que l'on ne peut détailler ici, mais cette exigence de pureté logique a au moins un avantage : elle empêche de construire une théorie de la confirmation subjectiviste, c'est-à-dire où la confirmation d'une théorie dépend de l'individu qui l'envisage et des détails particuliers de son existence. En effet, si l'on ne respecte pas cette exigence et que l'on admet que puissent jouer dans la confirmation d'une hypothèse des détails historiques tels que la date à laquelle elle a été formulée, ou la date à laquelle une preuve empirique a été découverte, on risque de se retrouver face à des situations telles que celle-ci :

Albert et Bernardo sont deux chercheurs appartenant à des laboratoires différents, l'un en France, l'autre en Espagne. En France le laboratoire d'Albert découvre le nouvel effet $\mathrm{E}$ en physique des particules, mais décide de garder l'information secrète tant qu'Albert n'a pas élaboré une théorie en rendant compte. Albert formule la théorie T. En Espagne, personne dans le laboratoire de Bernardo n'a aucune idée de ce qui se trame dans les laboratoires français, mais Bernardo, étudiant aussi la physique des particules, formule la même théorie T qu'Albert. Lorsque le laboratoire français rend publique sa découverte

1. Décernée par la Royal Academy à partir de 1877, cette médaille était considérée comme la plus haute distinction scientifique en chimie avant l'apparition des prix Nobel.

2. P. Lipton, Inference to the Best Explanation, Londres, Routledge, 1991, p. 134.

3. C. Hempel, « Studies in the logic of confirmation I», Mind, $\mathrm{n}^{\circ} 213,(1945)$, p. 5.

4. A. Musgrave, «Logical Versus Historical Theories of Confirmation », British Journal for the Philosophy of Science, $\mathrm{n}^{\circ} 25$ (1974), p. 2. 
de l'effet $\mathrm{E}$, on se rend compte que la théorie de Bernardo le prédisait, tandis que celle d'Albert l'accommodait. Il s'agit de la même théorie T mais si l'on est prédictiviste on doit admettre qu'elle est plus confirmée en deçà des Pyrénées qu'au-delà, et que la même théorie peut être plus ou moins confirmée selon que l'on est dans la peau d'Albert ou Bernardo.

La notion de prédiction ne permet donc pas de construire une théorie de la confirmation où le degré de validité empirique d'une théorie peut être mesuré objectivement, comme l'exigeait Hempel. Si elle permettait quelque chose, ce serait tout au plus de participer à une évaluation des degrés de croyance personnelle que chaque scientifique attribue aux théories.

Il apparut ainsi très tôt à ceux qui voulaient intégrer la notion de prédiction à la théorie de la confirmation que celle-ci amenait à violer l'exigence de pureté logique de la confirmation. Remarquons cependant dès à présent que l'on n'aboutit à cette conclusion que parce que la différence entre prédiction et accommodation ne repose que sur une distinction temporelle, chronologique, entre faits prédits et faits accommodés.

Mais le prédictivisme n'est pas qu'une thèse normative cherchant à définir quelles preuves devraient avoir le plus de poids dans la confirmation des hypothèses : c'est aussi et surtout une thèse descriptive cherchant à faire place au fait que les prédictions semblent avoir une grande importance dans la méthodologie et les pratiques des scientifiques puisque l'histoire des sciences semble montrer qu'elles ont joué un grand rôle dans l'acceptation ou le rejet de nouvelles théories.

Cependant, d'un point de vue historique, s'il est clair que les prédictions sont souvent citées a posteriori parmi les meilleures preuves, ce n'est pas pour autant qu'elles ont eu auprès des scientifiques une grande influence pour faire accepter ou rejeter de nouvelles hypothèses. Depuis la fin des années 1980 des historiens ont enquêté, documents à l'appui, sur ce rôle des prédictions dans l'acceptation de nouvelles théories. S. J. Brush ${ }^{5}$ a ainsi étudié systématiquement un grand nombre de cas historiques de succès prédictifs et en a conclu qu'ils n'ont pas plus joué dans l'acceptation de nouvelles théories que l'accommodation de faits déjà connus.

Dans le cas des prédictions de Mendeleïev, comme le remarque Eric Scerri, un des spécialistes actuels de l'histoire de la classification périodique des éléments, la médaille Davy n'a probablement pas été décernée au chimiste russe pour la prédiction de nouveaux éléments puisqu'il n'en est fait aucune mention dans le discours accompagnant la remise du prix ${ }^{6}$. De plus, Mendeleïev reçoit la médaille en même temps que le chimiste

\footnotetext{
5. S. Brush, «Prediction and Theory Evaluation : the case of light bending », Science, $\mathrm{n}^{\circ} 246$, (1989), «Prediction and Theory Evaluation: Subatomic particles », Rivista di Storia della Scienza, vol. 1, $\mathrm{n}^{\circ} 2$ (1993), "Theory and Experiment in the Quantum-Relativity Revolution », American Physical Society Meeting 2010.

6. Cf. E. Scerri et E. Worrall, «Prediction and the Periodic Table», Studies in History and Philosophy of Science, $\mathrm{n}^{\circ}$ 32, (2001), p. 415 où le discours est reproduit dans son intégralité.
} 
allemand Meyer, considéré comme le codécouvreur de cette classification bien qu'il n'ait pas réalisé autant de prédictions que Mendeleïev.

Enfin $^{7}$, ajoutons que dans la note à l'Académie des Sciences où le chimiste Lecoq de Boisbaudran annonce sa découverte du Gallium ${ }^{8}$, il ne fait aucune mention de Mendeleïev puisqu'il n'avait pas connaissance des prédictions de ce dernier. C'est Mendeleïev lui-même qui dans un courrier à l'Académie en $1875^{9}$ remarque que le Gallium est l'élément qu'il avait prédit en 1871. Lecoq de Boisbaudran n'a donc pas découvert le Gallium grâce à la prédiction de Mendeleïev, mais de manière indépendante et puisque cette découverte aurait pu être réalisée avant 1871 il est purement accidentel qu'elle compte comme une prédiction de la théorie de Mendeleïev et qu'elle la confirme.

Ainsi, les prédictions de la classification de Mendeleïev ne semblent pas avoir compté plus que les accommodations dans son adoption par les chimistes. Mais cela ne dépend à nouveau que du fait que l'on a longtemps cru pouvoir distinguer uniquement chronologiquement prédictions et accommodation et donc ne compter comme des prédictions de Mendeleïev que celles qui concernaient des éléments inconnus et non, par exemple, les prédictions qu'il a faites au sujet de certaines propriétés chimiques et physiques d'éléments déjà connus.

Aussi bien du point de vue logique qu'historique, il est donc apparu à ceux qui soutiennent le prédictivisme que si l'on veut fait droit à cette intuition partagée par les scientifiques et les non-scientifiques selon laquelle une prédiction est un important facteur de confirmation et d'acceptation d'une hypothèse, il faut réviser la définition des prédictions scientifiques et, paradoxalement, distinguer prédictions et accommodations sans faire appel à la différence temporelle entre faits prédits et faits accommodés.

Comme on va le voir, c'est parce que le débat sur le réalisme scientifique est arrivé à une conclusion similaire - mais non identique - que cette révision a eu lieu.

\section{Problèmes du critère prédictif de vérité}

Il y a au moins autant de versions différentes du réalisme scientifique que de philosophes qui soutiennent cette position (voire plus, puisque certains auteurs changent souvent de position). Cependant la plupart de ces versions s'accordent pour soutenir au moins cette revendication :

\footnotetext{
7. Scerri et Worrall dans leur article de 2001 développent de nombreux autres arguments historiques contre le scénario prédictiviste de la confirmation de la classification de Mendeleïev.

8. E. Lecocq de Boisbaudran, «Sur quelques propriétés du Gallium», Comptes-rendus hebdomadaires des séances de l'Académie des sciences, (1875), pp. 1100 sq.
}

9. D. Mendeleïev, «Remarques sur la découverte du Gallium », Comptes-rendus hebdomadaires des séances de l'Académie des sciences, (1875), pp. 969 sq. 
Les théories scientifiques matures ne sont pas seulement adéquates empiriquement mais aussi vraies ou approximativement vraies, c'est-à-dire qu'elles décrivent correctement les réalités non-observables auxquelles réfèrent leurs principaux termes théoriques.

L'adjectif «mature» vise à écarter les cas de théories scientifiques passées qui furent adéquates aux expériences alors connues mais en recourant à des entités fictives selon nos théories actuelles. On peut ainsi écarter la dynamique d'Aristote ou la chimie du phlogistique de Priestley de la liste des théories que l'on considère comme vraies et non simplement empiriquement adéquates.

Mais comment distinguer les théories matures décrivant approximativement l'ordre réel du monde de celles, immatures, qui ne font qu'imaginer des entités et des principes fictifs selon leurs besoins ? On ne peut clairement pas, sans risque de circularité, affirmer que les seules théories matures sont celles dont les entités sont conservées dans nos théories actuelles. Il faut donc trouver, comme le souligne John Worrall, «un critère raisonnablement précis et indépendant de maturité des théories $»^{10}$.

Selon lui ce critère peut être «lu comme dans un livre ouvert» dans le principal argument en faveur du réalisme scientifique : l'argument du miracle. Cet argument dû à Hilary Putnam est ainsi nommé car il consiste à affirmer que le réalisme est la seule philosophie qui ne fasse pas du succès de la science un miracle, puisqu'il serait extrêmement improbable que nos théories scientifiques soient adéquates aux phénomènes observables tout en étant fausses. On peut donc, du succès scientifique des théories, inférer que nos théories sont vraies ou approximativement vraies. Ainsi, pour qu'une théorie soit mature, il faut qu'elle soit couronnée de succès, et pour cela il faut, selon Worrall, qu'elle fasse plus qu'avoir des conséquences empiriques vraies (car on peut toujours utiliser une hypothèse ad hoc pour que cela soit le cas), il faut qu'elle réalise d'authentiques prédictions innovantes (genuine novel prediction). Un critère prédictif de maturité des théories, c'est-à-dire n'attribuant la maturité qu'aux théories réalisant de telles prédictions, est ainsi par conséquent aussi critère de leur vérité.

Mais adopter ce critère a aussi impliqué de distinguer prédictions et accommodations autrement que de manière chronologique. En effet, si l'on se contente de distinguer temporellement prédictions et accommodations (ce que Worrall ne fait pas) on fait entrer dans le club des théories matures de nombreuses théories que l'on considère aujourd'hui comme fausses. On peut par exemple déduire du système astronomique de Ptolémée la date de très nombreuses prédictions de conjonctions, d'éclipses, de rétrogradations planétaires, etc., bien avant qu'elles ne se produisent. De même les théories géologiques «catastrophistes», qui expliquaient la forme du paysage

10. J. Worrall, «Structural Realism : The Best of Both Worlds ? », Dialectica, n 43, (1989), p. 113. 
terrestre par des événements comme le Déluge biblique, auraient pu affirmer qu'il existe des fossiles marins en altitude ou dans des déserts avant leur découverte. Pourtant nous ne considérons plus que les épicycles et les catastrophes divines sont des entités ou des événements réels.

On a donc aussi rencontré dans le débat sur le réalisme scientifique la nécessité de réévaluer la définition de la notion de prédiction pour construire un critère prédictif de vérité qui ne soit pas trop large. Mais on peut remarquer dès à présent que le fait que l'on cherche une nouvelle définition des prédictions scientifiques en théorie de la confirmation et dans la question $\mathrm{du}$ réalisme scientifique n'implique pas pour autant que l'on soit à la recherche de la même définition dans ces deux domaines: il semble notamment que le succès prédictif requis pour affirmer la réalité des entités supposées par une théorie doit être bien plus fort et donc moins fréquent que celui requis uniquement pour la confirmer empiriquement.

Malgré cette différence, dont on montre plus loin les conséquences, on a vu apparaître au cours des années 2000 dans la littérature portant sur le réalisme scientifique et sur la théorie de la confirmation les mêmes questions presque standardisées qui constituent le problème spécifique des prédictions scientifiques et qui sont détaillées ci-dessous.

\section{Les problèmes spécifiques aux prédictions scientifiques}

On considère habituellement que la littérature moderne sur les prédictions scientifiques commence en théorie de la confirmation en 1974 avec l'article de Musgrave et 1989 dans le débat sur le réalisme scientifique avec l'article de John Worrall. Ce n'est qu'en 2004 cependant que Hitchcock et Sober ${ }^{11}$ firent explicitement le lien entre ces deux domaines et posèrent ce problème sous une forme aujourd'hui standard. Derrière ce problème apparemment unifié se cachent en réalité plusieurs questions :

a. Comment différencier prédiction et accommodation? C'est la question de la nature des prédictions ;

b. Les prédictions comptent-elles toujours plus que les accommodations ou ne comptent-elles que parfois plus, lorsqu'elles révèlent certaines qualités des théories qui les réalisent ? C'est la question de la valeur des prédictions.

En réalité, comme le remarquent Hitchcock et Sober, la question de la valeur des prédictions peut être scindée en deux :

b.1. Est-ce que les prédictions comptent toujours plus ou seulement parfois plus que les accommodations? C'est la différence entre un prédictivisme local et un prédictivisme global;

11. C. Hitchcock et E. Sober, «Prediction Versus Accommodation and the Risk of Overfitting », British Journal for the Philosophy of Science, $n^{\circ} 55$, (2004), p. 4. 
b.2. Est-ce que les prédictions sont intrinsèquement supérieures aux accommodations, ou ne sont-elles que symptomatiques d'autres vertus théoriques qui ont, elles, une influence directe sur la confirmation de la théorie ? C'est la différence entre un prédictivisme fort et un prédictivisme faible.

Non seulement ces différentes formes de prédictivisme peuvent se recouper - on peut soutenir une position prédictiviste globale et faible ou globale et forte - mais elles sont aussi relatives au type de distinction entre prédiction et accommodation que l'on soutient, c'est-à-dire à la réponse que 1'on donne à la question a.

En effet, on a vu qu'une distinction temporelle entre faits prédits et faits accommodés avaient des conséquences fâcheuses, et c'est pour cela que deux autres types de distinctions (et donc de définitions des prédictions) ont été développées :

- une distinction heuristique : les faits prédits sont ceux qui n'ont pas servi à construire la théorie tandis que les faits accommodés sont ceux qui ont été utilisés dans la construction de la théorie et qu'elle contient donc à l'origine. Cette thèse fut initialement proposée par Elie Zahar ${ }^{12}$ puis défendue et complexifiée par John Worrall ${ }^{13}$;

- une distinction théorique: les faits prédits par une théorie sont ceux qu'aucune autre théorie rivale ne permet de déduire. Cette thèse fut défendue par Imre Lakatos et Alan Musgrave ${ }^{14}$.

Tenter de résoudre le problème spécifique des prédictions scientifiques consiste, depuis l'article d'Hitchcock et Sober, à argumenter en faveur d'une des multiples combinaisons de positions possibles et à montrer qu'elle respecte les deux conditions suivantes :

1. elle ne produit pas une théorie de la confirmation subjectiviste ;

2. elle rend compte de certains cas d'histoire des sciences paradigmatiques: les prédictions du système Copernicien, celles de la théorie de la gravitation de Newton, de l'optique de Fresnel, de la classification de Mendeleïev et de la Relativité Générale.

Si la thèse ainsi soutenue respecte ces deux conditions, on peut alors déterminer si les prédictions réussies constituent ou non un succès empirique

12. E. Zahar, «Why did Einstein's Programme Supersede Lorentz's? », British Journal for the Philosophy of Science, $\mathrm{n}^{\circ} 24$ (1973).

13. J. Worrall, «Fresnel, Poisson and the white spot: The role of successful predictions in the acceptance of scientific theories ", in The Uses of Experiment: Studies in the Natural Sciences, D. Gooding, T. Pinch et S. Schaffer (eds.), Cambridge, Cambridge University Press, 1989 ; et "Theory-Confirmation and History », in Rationality and Reality ; conversations with Alan Musgrave, C. Cheyne and J. Worrall (eds.), Kluwer, 2005.

14. I. Lakatos, "Falsification and the Methodology of Scientific Research Programmes », in Criticism and the Growth of Knowledge, I. Lakatos and A. Musgrave (eds.), Cambridge University Press, 1970. 
suffisant pour soutenir l'argument du miracle, et donc exporter ces résultats dans le débat sur le réalisme scientifique.

L'objectif ici est de présenter maintenant une des solutions qui a été proposée à ce problème, certaines modifications que l'on pourrait lui apporter et enfin de montrer que cette exportation de résultats vers le débat sur le réalisme scientifique a pu entraîner certaines confusions.

\section{Solutions, objections et perspectives}

L'article d'Hitchcock et Sober n'est pas uniquement fondateur dans sa manière de poser le problème, mais aussi dans sa manière de l'aborder et d'y répondre. Il a donc ouvert de nombreuses perspectives que l'on va tâcher d'exploiter ici.

\section{Prédictions, accommodation et risque de sur-ajustement}

Eliott Sober, avec Malcom Forster ${ }^{15}$ dans un premier temps, puis Christopher Hitchcock dans un second, a remarqué plusieurs fois que malgré l'attention portée à l'histoire des sciences dans le débat sur les prédictions scientifiques, peu d'exemples de processus prédictifs réels y sont discutés. Il s'appuie pour sa part sur un domaine des mathématiques appliquées qui a pour tâche de résoudre des problèmes de prédictions : l'analyse prédictive en statistiques.

Le but de cette analyse, qui est une forme d'analyse régressive, est de trouver à partir d'un ensemble de données $D_{1}$, le modèle qui permettra de prédire le mieux un autre ensemble de données $D_{2}, D_{1}$ et $D_{2}$ étant des échantillons tirés de la même population. Par modèle on entend ici une hypothèse ayant au moins un paramètre dit "libre », c'est-à-dire deux symboles dont on règle la valeur en fonction des données auxquelles on veut s'ajuster. Si les données consistent en un ensemble de valeurs d'une variable $\mathrm{Y}$ en fonction d'une variable $\mathrm{X}$ (par exemple le volume d'un gaz en fonction de sa température) on peut représenter $D_{1}$ sous la forme de points dans un repère (cf. figure 1 ), et le modèle à trouver est une courbe dont l'équation doit contenir au moins un paramètre à ajuster. Le modèle linéaire $\mathrm{M}$ par exemple est une ligne dont l'équation est $\mathrm{Y}=\mathrm{aX}+\mathrm{b}$ (cf. figure 2). Ce modèle a deux paramètres dits libres : a et $b$, le premier déterminant la pente de la droite et le deuxième sa hauteur à l'origine.

15. M. Forster et E. Sober, « How to Tell when Simpler, More Unified, or Less Ad Hoc Theories will Provide More Accurate Predictions », British Journal for the Philosophy of Science, $\mathrm{n}^{\circ} 45,(1994)$. 


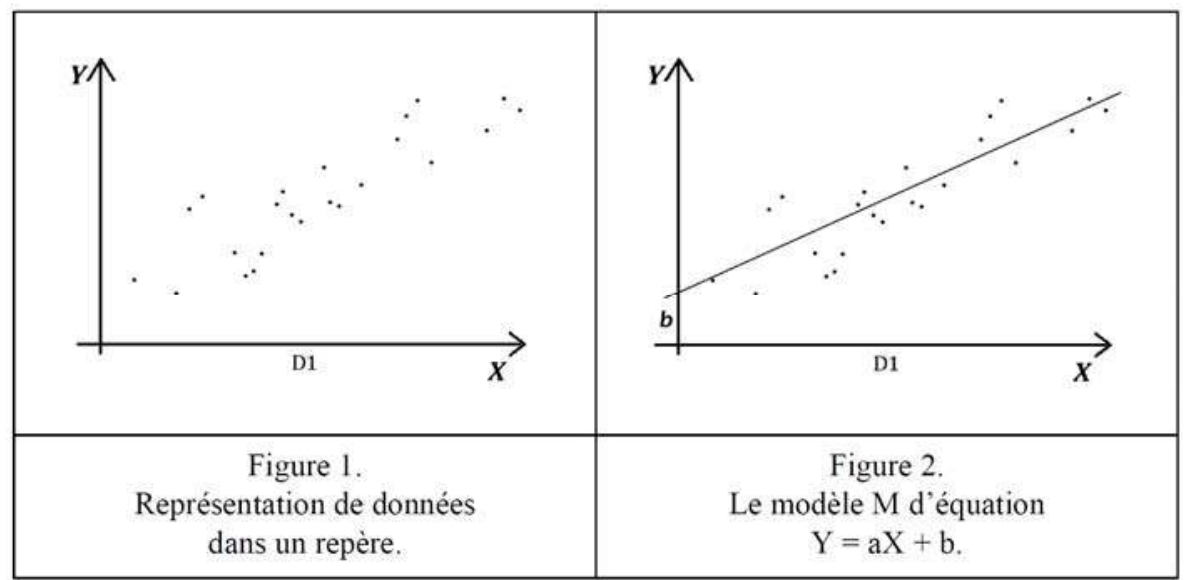

Un modèle hyperbolique du type $\mathrm{Y}=\mathrm{aX} \mathrm{X}^{2}+\mathrm{bX}+\mathrm{c}$ par exemple serait considéré comme plus complexe que $\mathrm{M}$ puisqu'il aurait trois paramètres à ajuster : $a, b$ et $c$.

Hitchcock et Sober peuvent donc définir les faits accommodés d'un point de vue heuristique en considérant que l'ensemble des données utilisé pour fixer la valeur des paramètres libres est accommodé, tandis que les autres sont prédits.

Nous sommes pour l'instant face à un problème d'ajustement de courbe (curve-fitting): il s'agit de trouver la courbe qui passe au plus près des points, c'est-à-dire qui est la plus précise, la plus adéquate aux données. On mesure cet éloignement en calculant la vraisemblance (likelihood) de la courbe, qui correspond à la probabilité d'obtenir les données observées selon la valeur des paramètres libres ${ }^{16}$.

Or on peut prouver que l'on peut réduire cet éloignement entre données et courbes à zéro, puisqu'il existe, pour toute répartition de $n$ points de données dans un repère, une fonction polynomiale de degré n-1 (c'est-à-dire de la forme $Y=a_{n-1} X^{n-1}+a_{n-2} X^{n-2}+\ldots+a_{2} X^{2}+a_{1} X+a_{0}$ ) qui passe par chacun des $n$ points de donnée. Autrement dit, on peut toujours trouver un modèle $\mathrm{M}^{\prime}$ dont la précision est maximale, même s'il est très complexe en raison de ses $n$ paramètres libres.

Mais lorsqu'on construit de tels modèles à partir d'un ensemble $\mathrm{D}_{1}$ de données et qu'on essaye ensuite de prédire avec eux un autre échantillon $\mathrm{D}_{2}$ on se rend compte qu'ils ont une faible vraisemblance par rapport aux données $\mathrm{D}_{2}$ - c'est-à-dire une mauvaise précision vis-à-vis des données prédites.

16. On manipule souvent, pour des raisons pratiques, le logarithme népérien de la vraisemblance noté « $\ln ($ vraisemblance $[\mathrm{M}]) »$. 


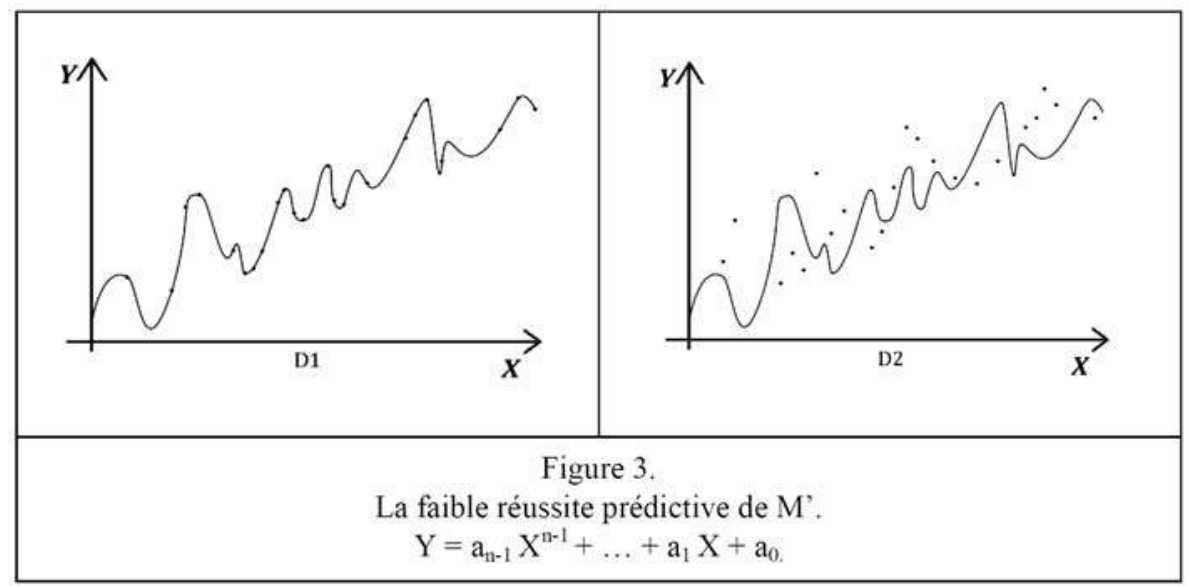

\section{Comme le remarquent Hitchcock et Sober :}

Pour les statisticiens, les mathématiciens appliqués et autres scientifiques qui construisent fréquemment des modèles pour tirer du sens de données, c'est un fait bien confirmé que les modèles les plus complexes sont souvent peu efficaces pour prédire de nouvelles données ${ }^{17}$.

Qu'est-ce qui explique ce fait ? La courbe du modèle M', en " collant » aux données $\mathrm{D}_{1}$ risque d'être sur-ajustée (overfitting), c'est-à-dire de chercher à rendre compte avec précision de variations qui ne sont qu'accidentelles. En effet, il est tout à fait possible que la dispersion des données ne dépendent pas que de la relation que la grandeur $\mathrm{X}$ entretient avec la grandeur $\mathrm{Y}$ mais aussi d'un certain nombre de petites causes indépendantes les unes des autres et de $\mathrm{X}$ et $\mathrm{Y}$ dont les effets varient à chaque fois que l'on récolte des données.

Prenons l'exemple de mesures de la pression d'un gaz en fonction de sa température. Ces deux grandeurs sont, selon la théorie cinétique des gaz, proportionnelles. Mais lors des mesures de l'ensemble de données $\mathrm{D}_{1}$ un certain nombre de petites causes d'erreurs comme la chaleur du thermomètre ou les variations de la pression atmosphérique risquent fort de faire varier le résultat de ces mesures, causant une dispersion des résultats ${ }^{18}$ autour de la droite d'équation $\mathrm{Y}=\mathrm{aX}$ que devrait suivre la relation entre pression et température.

17. C. Hitchcock et E. Sober, «Prediction Versus Accommodation and the Risk of Overfitting », op. cit., p. 11.

18. On peut considérer sous certaines conditions que cette dispersion est une courbe de Gauss, c'est-à-dire une distribution normale, centrée sur une valeur pouvant être considérée comme la «vraie » valeur. Celle-ci correspond à un signal dont la perturbation serait le bruit. Le risque de sur-ajustement correspond donc au risque de prendre le bruit pour le signal. 
Ainsi, si l'on ajustait un modèle $M$ ' à cet ensemble de données $D_{1}$ et que l'on répétait ensuite la même expérience pour obtenir un échantillon $\mathrm{D}_{2}$, il y a très peu de chances que ces petites causes agissent de la même manière et créent la même dispersion que celle à laquelle a été ajustée $\mathrm{M}^{\prime}$ : on se retrouverait donc dans la situation de la figure 3. Pourtant, puisque les petites causes de perturbation agissent de manière indépendante, les données de $\mathrm{D}_{2}$ seront toujours dispersées autour d'une droite $\mathrm{Y}=\mathrm{aX}$. Un modèle linéaire simple comme $\mathrm{M}$ conserverait donc à peu près la même précision sur $\mathrm{D}_{1}$ et sur $\mathrm{D}_{2}$ (cf. figure 4) tandis que le modèle complexe $\mathrm{M}^{\prime}$, en maximisant sa précision sur $\mathrm{D}_{1}$, « est trop sensible aux idiosyncrasies des données qui n'ont que peu de chance de réapparaître dans d'autres échantillons $\gg{ }^{19}$.

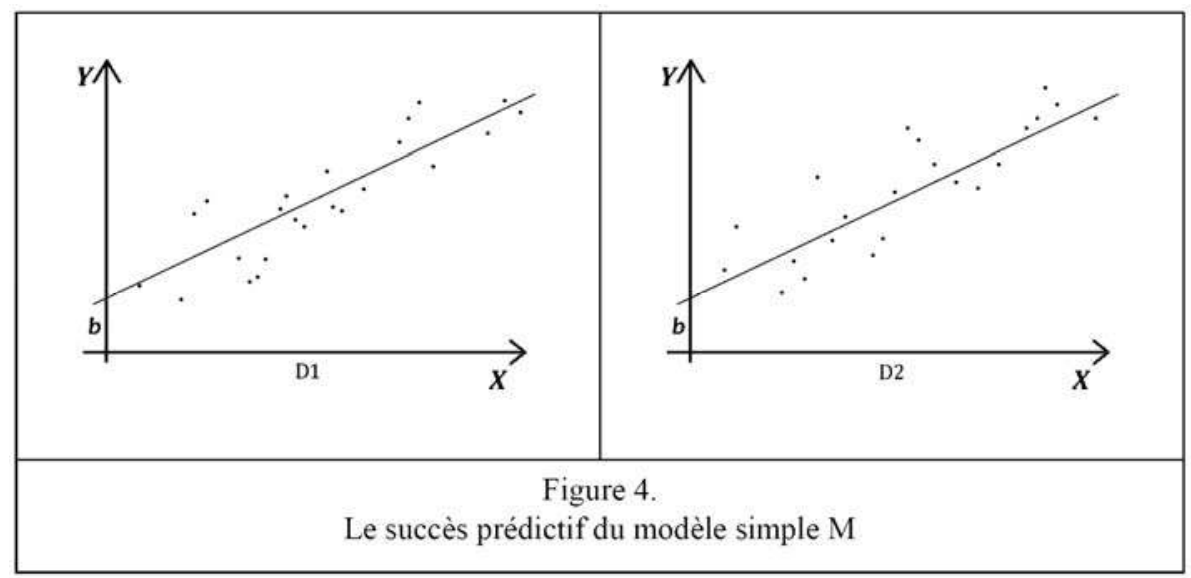

Puisque la simplicité d'un modèle évite le risque de sur-ajustement, lorsqu'on choisit un modèle il faut équilibrer son ajustement aux données par sa simplicité si l'on veut maximiser sa précision prédictive. Hitchcock et Sober proposent de reprendre le résultat du statisticien Akaike qui en $1973^{20}$ a montré que l'on pouvait estimer ainsi la précision prédictive du modèle $\mathrm{M}$ :

- estimation (non biaisée) de la précision prédictive de $\mathrm{M}=\ln ($ vraisemblance $\mathrm{L}(\mathrm{M}))-\mathrm{k}$,

- où - $M$ est un modèle,

- $L(M)$ est l'ajustement des paramètres ajustables de ce modèle qui maximalise sa vraisemblance aux données déjà connues,

- In(vraisemblance de $L(M)$ ) représente la mesure de son ajustement aux données disponibles,

19. C. Hitchcock et E. Sober, «Prediction Versus Accommodation and the Risk of Overfitting », op. cit., p. 11.

20. H. Akaike, «Information Theory as an Extension of the Maximum Likelihood Principle », in Second International Symposium on Information Theory, B. Petrov and F. Csaki (eds.), Budapest, Akademiai Kiado, 1973. 
- $k$ est le nombre de paramètres de l'équation de L(M), et représente donc la complexité de M.

Lorsqu'on calcule pour un modèle donné la valeur de cette équation habituellement appelée Critère d'Information d'Akaike (AIC), on évalue son " score AIC » mesurant son équilibre entre simplicité et précision vis-à-vis des données accommodées. Akaike a montré que le score AIC d'un modèle correspondait aussi à la précision moyenne maximale des prédictions de ce modèle. Autrement dit, puisque l'on peut évaluer le score AIC d'un modèle uniquement en considérant son ajustement à un seul échantillon, cette équation d'Akaike a la propriété très intéressante de permettre de mesurer la précision prédictive d'un modèle sans avoir à réaliser et à tester de prédictions. Dans notre exemple on pourrait, à partir de cette formule, calculer le score AIC de $\mathrm{M}$ et de $\mathrm{M}$ ' uniquement en regardant leur nombre de paramètres ajustables et leur vraisemblance sur $\mathrm{D}_{1}$, c'est-à-dire avant même de les comparer à $\mathrm{D}_{2}$.

L'AIC permet à Hitchcock et Sober de montrer que même si l'on se donne pour objectif de sélectionner les modèles ayant la plus grande précision prédictive, on ne peut soutenir un prédictivisme global puisqu'il existe des cas où l'on devrait préférer un modèle accommodant des données à un autre les prédisant. En effet, si jamais un modèle accommode l'ensemble de données $\mathrm{D}^{21}$ en essayant de maximiser non son ajustement aux données mais son score AIC, c'est-à-dire en respectant l'impératif selon lequel toute perte de simplicité doit être compensée par un gain très important en précision, alors il n'y a pas de raison de considérer qu'il est inférieur à un modèle accommodant $\mathrm{D}_{1}$ et prédisant $\mathrm{D}_{2}$. Au contraire, son score AIC ne peut être qu'égal ou supérieur à ce modèle confirmé par une prédiction, puisqu'il aura le même nombre de paramètres que celui-ci mais une vraisemblance supérieure ou égale. Dans ce cas-là, donc, une accommodation confirme mieux une théorie ou un modèle qu'une prédiction.

Le travail de Sober, Hitchcock et Forster, en plus de traiter des problèmes liés à la définition et à la valeur de la simplicité et de la précision, permet de répondre aux problèmes spécifiques des prédictions scientifiques :

a. Si l'on considère qu'accommoder des données consiste à fixer des paramètres en ajustant un modèle à des données, Hitchcock et Sober ont une définition heuristique de la prédiction : peu importe la date à laquelle ont été récoltées les données $\mathrm{D}$, seul importe de savoir si l'ensemble de ces données ou juste un de ses sous-ensembles a été utilisé pour ajuster les paramètres du modèle ou de la théorie à confirmer, c'est-à-dire dans sa construction ;

b.1. Hitchcock et Sober développent une version locale du prédictivisme puisqu'ils discriminent (et donnent le moyen de discriminer) les cas où les prédictions sont supérieures aux accommodations des cas où elles leurs sont inférieures ;

21. Avec $\mathrm{D}=\mathrm{D}_{1} \cup \mathrm{D}_{2}$. 
b.2. Hitchcock et Sober n'encourent pas pour autant le risque de subjectivisme car ils soutiennent un prédictivisme faible. En effet, selon eux, même si l'on se donne pour but, en sélection de modèles, la précision prédictive, les prédictions ne sont pas intrinsèquement importantes mais symptomatiques d'une certaine vertu théorique: un bon équilibre en précision et simplicité. Cette vertu est une propriété intrinsèque aux théories, et ne viole donc pas l'impératif de pureté logique de la confirmation.

Hitchcock et Sober n'abordent pas d'exemples paradigmatiques de 1'histoire des sciences dans leur article de 2004, mais dans un autre article Forster et Sober comparent le score AIC du système Copernicien et du système Ptoléméen, montrant qu'il faudrait que la vraisemblance de ce dernier soit plus de 485 millions de fois supérieure à celle du système Copernicien pour que son score lui soit supérieur ${ }^{22}$.

Ils examinent par contre en profondeur les conséquences de leur position sur la notion de prédiction innovante et sur l'argument du miracle. Selon eux, on peut très bien obtenir une courbe qui ne soit pas la "vraie " courbe, au sens de la courbe représentant la relation entre les grandeurs dont la perturbation serait responsable de la distribution des données, mais qui ait un bon score AIC, c'est-à-dire qui puisse conserver une bonne précision même par rapport à des échantillons auxquels elle n'a pas été ajustée. Autrement dit, le succès prédictif n'est pas suffisant pour juger de la maturité et donc de la vérité d'une théorie.

\section{Prédictions et capacité prédictive}

Il ne serait pas possible ici de décrire l'ensemble des nombreuses réponses et objections suscitées par l'article de Hitchcock et Sober. Il s'agit plutôt ici de reprendre leurs résultats et de montrer que l'on peut les interpréter différemment afin de soutenir non seulement une version forte $\mathrm{du}$ prédictivisme mais aussi critiquer leurs conclusions sur la question du réalisme scientifique.

Kit Patrick $^{23}$ a fait remarquer que l'on ne violerait pas la pureté logique de la confirmation si l'on ne considérait plus les prédictions réellement réalisées mais la capacité des théories à les réaliser, c'est-à-dire leur capacité prédictive. En effet, la capacité d'une théorie à prédire des phénomènes étant une propriété de cette théorie, on ne viole pas l'impératif selon lequel seules les propriétés des théories, des preuves et leurs relations doivent être prises en compte pour évaluer la confirmation d'une théorie. On peut donc adopter sans risque de produire une théorie subjectiviste de la

22. M. Forster et E. Sober, «How to Tell when Simpler, More Unified, or Less Ad Hoc Theories will Provide More Accurate Predictions », op. cit., p. 14.

23. K. Patrick, « Prediction, Predictability and Confirmation », (2011), à paraître. 
confirmation un prédictivisme fort quant à la capacité prédictive, c'est-à-dire considérer que la capacité prédictive des théories a en soi de l'importance et n'est pas qu'un symptôme d'une autre vertu théorique.

On dit d'une théorie qu'elle a une bonne capacité prédictive lorsque d'une part elle permet de prédire des phénomènes précisément et d'autre part lorsqu'elle permet de prédire une large gamme de phénomènes différents. En effet, on préférera à une théorie permettant de prédire la position d'une planète à cinq jours près une autre qui la prédit à un jour près, et à une théorie permettant uniquement de prédire la position de cette planète une autre qui prédit aussi son volume et sa masse. Autrement dit, la capacité prédictive d'une théorie a deux dimensions :

- l'étendue des phénomènes et grandeurs différents pouvant être prédits : c'est la dimension extensive de la capacité prédictive ;

- la précision des prédictions réalisées pour chaque type de phénomène et de grandeur: c'est la dimension intensive de la capacité prédictive.

Mais comment parler de capacité prédictive d'une théorie sans parler en même temps de prédictions réellement réalisées ? Comment savoir si une théorie $T$ peut prédire un phénomène sans réaliser un certain nombre de prédictions à partir de $\mathrm{T}$ puis attendre de voir si elles se réalisent ou non ? Existe-t-il un moyen de se passer des tests des prédictions d'une théorie pour évaluer sa capacité à faire des prédictions?

On a montré ci-dessus que le résultat d'Akaike avait précisément ceci de remarquable qu'il permettait de mesurer la précision prédictive d'un modèle en ne considérant que son nombre de paramètres et son ajustement aux données qu'il accommode, c'est-à-dire sans avoir à réaliser et à tester de prédictions. Si l'on prend le point de vue de cette capacité prédictive des théories en considérant que le score AIC d'un modèle évalue une des dimensions de cette capacité, on peut réinterpréter les exemples de Hitchcock et Sober pour montrer que ce sont toujours les modèles ayant la plus grande capacité prédictive qui sont choisis.

Lorsqu'on préfère par exemple un modèle ayant prédit une partie des données à un modèle n'ayant réalisé que des accommodations, on préfère le modèle prédictif uniquement parce que cette prédiction est un signe du fait que ce modèle a probablement un bon score AIC car il n'est pas sur-ajusté, au contraire du modèle accommodant qui encourt ce risque. La prédiction réellement réalisée n'a d'importance qu'en tant qu'indice que le modèle prédictif a une meilleure capacité prédictive que son rival.

Il en va de même, dans les cas où les accommodations sont supérieures aux prédictions selon Hitchcock et Sober. Dans ces cas on a à faire à un modèle accommodant construit pour éviter le risque de sur-ajustement. Il a la même étendue prédictive que le modèle prédictif et ne s'en distingue que par sa précision prédictive mesurée par ce score AIC : on préfère donc le modèle ayant la plus grande capacité prédictive. Même si c'est un modèle accommodant les données qui est sélectionné, c'est bien en réalité la 
capacité prédictive en elle-même qui est valorisée et choisie. Comme le remarquent Hitchcock et Sober eux-mêmes, "l'innovation d'Akaike n'était pas seulement de montrer comment l'on pouvait estimer la précision prédictive mais aussi de définir la précision prédictive comme un objectif dans la sélection de modèle $»^{24}$, c'est-à-dire d'en faire un but en soi en statistique.

Ainsi, si Hitchcock et Sober soutiennent une forme de prédictivisme faible et local vis-à-vis des prédictions réellement réalisées, puisqu'ils présentent la maximisation du score AIC d'un modèle comme un but en soi et que ce score AIC représente une des dimensions de la capacité prédictive des théories, leur conception est compatible avec un prédictivisme fort et global vis-à-vis de la capacité prédictive des théories : peu importe qu'une théorie ait réalisé des prédictions ou des accommodations tant que celles-ci témoignent d'une forte capacité prédictive car cette propriété est toujours préférée et est préférée pour elle-même.

\section{Une conception unifiée des vertus scientifiques}

On pourrait objecter que, selon Hitchcock et Sober, ce n'est pas tant le score AIC d'un modèle qui est sélectionné que l'équilibre entre simplicité et précision. C'est cette vertu d'équilibre qui est en soi à valoriser lorsqu'on cherche à évaluer la confirmation d'une théorie ou à choisir entre plusieurs théories.

Mais on pourrait tout autant se demander s'il y a un sens à chercher, qui de la capacité prédictive ou de l'équilibre entre précision et simplicité doit être considérée comme un symptôme de l'autre si l'une ne va pas sans l'autre. Cette question a cependant un intérêt qui est de déterminer quelle conception des vertus théoriques adopter : est-ce qu'une bonne théorie est une théorie qui regroupe un certain nombre de vertus - voire une seule - et en sacrifier d'autres ou est-ce qu'une bonne théorie doit les posséder toutes en une certaine proportion?

Peter Lipton ${ }^{25}$ par exemple, considère qu'il existe, en théorie de la confirmation, certaines vertus attribuables aux preuves empiriques et d'autres attribuables aux hypothèses. Ainsi, pour que des expériences confirment le mieux possible une théorie, il faut qu'elles soient à la fois précises, nombreuses, variées, bien contrôlées et répétables. On considère aussi, toutes choses égales par ailleurs, qu'une hypothèse est d'autant mieux confirmée qu'elle est simple, qu'elle unifie de nombreux domaines différents et qu'elle est en accord avec d'autres hypothèses déjà admises. Il y a une

24. C. Hitchcock et E. Sober, «Prediction Versus Accommodation and the Risk of Overfitting », op. cit., p. 12.

25. P. Lipton, «Testing Hypotheses: Prediction and Prejudice», Science, n 307 (2005), p. 220. 
certaine tension entre les vertus expérimentales de précision, de quantité et de variété et la vertu de simplicité des hypothèses. Les scientifiques, s'ils ont à accommoder des données variées, précises et nombreuses, peuvent être tentés de sacrifier la simplicité des hypothèses pour produire une théorie extrêmement complexe calquée sur ces données, comme dans les cas du surajustement de modèles statistiques ou ceux que Lipton nomme les cas de tricheries (fudges) par adjonction d'hypothèses ad hoc.

Dans cette perspective s'assurer qu'une théorie est capable de prédire des phénomènes, c'est s'assurer qu'on a pu concilier des vertus théoriques (de simplicité) et des vertus expérimentales (de précision). La capacité prédictive occupe parmi les vertus théoriques la place que Platon donne dans le Gorgias (509c) à la tempérance ( $\sigma \omega ́ \varphi \rho \omega v)$ parmi les vertus morales : à la fois une vertu qui résulte de l'équilibre des autres (du courage, de l'intelligence, de l'humilité, etc.) et une vertu sans laquelle les autres sont inutiles car prenant le pas les unes sur les autres elles risquent de mener à l'excès et à l'hubris.

Le prédictivisme fort revient donc à reconnaître que la capacité prédictive a une place particulière parmi les vertus théoriques : elle assure que l'on a affaire à une théorie qui s'applique bien à l'expérience, c'est-àdire qui repose sur un grand nombre de preuves expérimentales différentes sans pour autant avoir recours à des procédés ad hoc. Cela confirme l'intuition exposée en introduction, que les réussites prédictives représentent d'importants succès scientifiques, c'est-à-dire des cas paradigmatiques des relations entre théories et expériences valorisés dans la méthodologie et les pratiques scientifiques.

\section{Analyse prédictive et réalisme scientifique}

Si les analyses de Hitchcock et Sober ouvrent de nombreuses portes en théorie de la confirmation, il n'est pas sûr qu'il en aille de même pour la question du réalisme scientifique, car l'analyse statistique prédictive à laquelle ils font référence ne concerne pas les prédictions innovantes dont il est question dans ce débat.

En effet, lorsque John Worrall a proposé de prendre les prédictions innovantes comme critère pour éliminer les théories scientifiques immatures, il avait un type bien particulier de prédiction en tête :

Ces théories manifestent normalement une faible prédictivité [predictiveness] car, Popper ou non, les scientifiques généralisent par induction les résultats d'expérimentations bien contrôlées qui ont jusqu'à maintenant toujours eu les mêmes résultats. Mais le succès de telles manœuvres inductives, bien que sans doute miraculeux en lui-même, ne parle pas en faveur de la probable vérité d'une quelconque théorie explicative. Le type de succès prédictif qui semble clarifier l'intuition sous-jacente à l'argument du miracle 
est une forme bien plus forte, bien plus frappante de succès prédictif. Dans ce cas de succès prédictif fort, ce n'est pas seulement une nouvelle instance d'une vieille généralisation empirique qui découle d'une théorie mais une généralisation empirique parfaitement nouvelle, et qui vient à être confirmée ${ }^{26}$.

Ces prédictions de généralisations empiriques «parfaitement nouvelles » sont-elles celles qui sont évaluées par le résultat d'Akaike ? Non, car celui-ci est restreint aux problèmes d'interpolation où l'on examine la capacité prédictive d'un modèle uniquement pour une certaine gamme de valeur des variables. Comme on l'a dit, l'AIC permet d'inférer à partir de l'ajustement d'un modèle à un échantillon $\mathrm{D}_{1}$ son ajustement à un échantillon $\mathrm{D}_{2}$ si et seulement si cet échantillon est tiré de la même population que $D_{l}$. Il ne permet donc pas d'évaluer la capacité d'un modèle à prédire comment se comportent des grandeurs les unes par rapport aux autres au-delà des limites de ce que l'on a déjà observé, car dans ce cas on a à faire à un problème dit d'extrapolation, où l'on cherche par exemple à déterminer la relation entre des variables $\mathrm{X}$ et $\mathrm{Y}$ au-delà des valeurs de $\mathrm{X}$ déjà observées dans les échantillons à notre disposition.

Or ces problèmes d'extrapolation sont précisément ceux qui peuvent mettre au jour de nouvelles généralisations empiriques et de nouveaux phénomènes : c'est par exemple en faisant varier théoriquement la densité des corps au-delà de celles que l'on a pu observer que l'on a défini la densité-limite, dite de "Schwarzshild», d'un corps générant un champ gravitationnel dont même les photons ne peuvent s'échapper: un trou noir. Ce phénomène est une conséquence de la Relativité générale mais n'a pas été utilisé dans la construction de celle-ci ni "observé » avant les années 1970. Il s'agit donc bien d'un phénomène nouveau à la fois temporellement et heuristiquement, prédit par la Relativité générale. Toutes les découvertes de ce type n'auraient pu avoir lieu si l'on s'était restreint aux cas où l'on ne prédit que des données sur les mêmes valeurs des variables que les données ayant servi à accommoder une théorie.

Mais même s'il était possible de modifier l'équation d'Akaike pour traiter les problèmes d'extrapolation, cela ne suffirait pas pour rendre compte de ces prédictions de phénomènes nouveaux. En effet, si le critère d'Akaike est une bonne manière d'évaluer la précision prédictive des modèles ou des théories, il ne permet pas de définir et mesurer leur étendue prédictive, c'est-à-dire leur capacité à prédire de nombreuses classes de phénomènes différents. Or c'est cette dimension extensive de la capacité prédictive qui est mise en jeu lorsqu'on parle de nouvelles catégories de phénomènes prédites par une théorie, et non la dimension intensive de la capacité prédictive qui elle est évaluable par l'AIC.

Ainsi, la portée des arguments de Hitchcock et Sober sur la question du réalisme scientifique est limitée, car ils ne parlent tout simplement pas du même type de prédictions que ceux qui soutiennent l'argument du miracle.

26. J. Worrall, « Structural Realism : The Best of Both Worlds ?», op. cit., p. 114. 
Les prédictions de modèles sont qualitativement différentes des prédictions théoriques. Ce n'est pas parce que le problème de la confirmation et celui du réalisme scientifique se sont rejoints sur cette notion de prédiction qu'il faut considérer que celle-ci forme un bloc monolithique sans différences, et il faut prendre l'expression " prédiction de phénomènes nouveaux » au sérieux comme désignant une espèce bien particulière de prédictions.

\section{Conclusion}

Les deux racines du problème spécifique des prédictions scientifiques ont pu amener à penser que sa solution pourrait se faire en n'examinant pas la diversité des prédictions et des notions qui y sont reliées. Cependant, on ne peut faire l'économie d'un certain nombre de distinctions, entre prédictions de modèles et prédictions théoriques, précision prédictive et étendue prédictive, prédictions innovantes et prédictions classiques, prédictions scientifiques et prédictions pseudo-scientifiques, etc. C'est à ce prix seulement que l'on peut croiser sans les confondre les différentes problématiques qui font intervenir les prédictions : théorie de la confirmation des théories, débat sur le réalisme scientifique, mais aussi théorie de l'explication, étude du changement théorique, de la sous-détermination empirique et plus généralement de toutes les différentes facettes des rapports entre théorie et expérience. 